\title{
The Millimeterwave Spectrum of Four Rare Ketene Isotopomers
}

\author{
Antonio Guarnieri \\ Technische Fakultät - Hochfrequenztechnik, Institut für Physikalische Chemie der Universität Kiel, \\ D-24143 Kiel, Germany
}

Reprint requests to Prof. A. G.; E-mail: ag@tf.uni-kiel.de

Z. Naturforsch. 60a, 619-628 (2005); received May 17, 2005

The pure rotational spectra in the ground vibrational state of $\left(1,2-{ }^{13} \mathrm{C}\right)$ ketene, $\mathrm{H}_{2}{ }^{13} \mathrm{C}={ }^{13} \mathrm{CO}$, $\left(\mathrm{D}_{2}, 1{ }^{13} \mathrm{C}\right)$ ketene, $\mathrm{D}_{2} \mathrm{C}={ }^{13} \mathrm{CO},\left(\mathrm{D}_{2}, 2-{ }^{13} \mathrm{C}\right)$ ketene, $\mathrm{D}_{2}{ }^{13} \mathrm{C}=\mathrm{CO}$, and $\left(\mathrm{D}_{2},{ }^{18} \mathrm{O}\right)$ ketene, $\mathrm{D}_{2} \mathrm{C}=\mathrm{C}^{18} \mathrm{O}$, have been observed in the frequency region $200-350 \mathrm{GHz}$. All the spectral lines have been measured in natural abundances with a source modulated millimeterwave spectrometer.

From the measured R-branch transitions a set of rotational and centrifugal distortion constants for each isotopomer could be derived, using the Watson S-reduction formalism. Further, the rotational spectra of the two isotopomers (4,5-D)ketene, $\mathrm{D}_{2} \mathrm{CCO}$, and (4-D)ketene, DHCCO, which were already measured several years ago, have been extended to higher $J$-values and higher frequencies, as it is the case for all investigated isotopomers of this work. As a result of these studies a calculation of a mass-dependent structure will be the topic of a next paper.

Key words: Rotational Spectra; Ketene; Isotopomers; Structure.

\section{Introduction}

Ketene is significant as a simple asymmetric top molecule for spectroscopy, theoretical chemistry and reaction dynamics. Papers [1-3] contain recent reviews summarizing much of the work of the last 55 years.

Few years ago, Watson et al. [4] presented a valuable method, the $\mathrm{r}_{\mathrm{m}}$-method, to relate the ground state and the equilibrium moments of inertia of a molecule.

They analysed the up to that time observed moments of inertia of six isotopic ketenes to obtain a corresponding $r_{m}$-structure, but due to the reduced number of available isotopomers compared with the number of parameters to be determined, they got values for parameters not completely satisfactory.

In the mean time the spectrum of the monosubstituted isotopic species in its ground vibrational state has been subject to increasingly refined analysis [1], and further the spectrum of $\left({ }^{17} \mathrm{O}\right)$ ketene has also been measured [5]. The present paper is concerned with millimeterwave spectra of rare species containing $(1,2-$ $\left.{ }^{13} \mathrm{C}\right)$ ketene, $\mathrm{H}_{2}{ }^{13} \mathrm{C}^{13} \mathrm{CO},\left(\mathrm{D}_{2}, 1-{ }^{13} \mathrm{C}\right)$ ketene, $\mathrm{D}_{2} \mathrm{C}^{13} \mathrm{CO}$, $\left(\mathrm{D}_{2}, 2-{ }^{13} \mathrm{C}\right)$ ketene, $\mathrm{D}_{2}{ }^{13} \mathrm{CCO}$ and $\left(\mathrm{D}_{2},{ }^{18} \mathrm{O}\right)$ ketene, $\mathrm{D}_{2} \mathrm{CC}^{18} \mathrm{O}$.

To my knowledge no spectra of these last four isotopomers have been reported earlier. The observed spectra are sufficient to supply also an acceptable value for the rotational constant $A$ in agreement with the values obtained for all the other species investigated hitherto.

\section{Experimental}

Ketene was prepared by vacuum pyrolysis of acetic anhydride at $850{ }^{\circ} \mathrm{C}$ and perdeutereted ketene from $\mathrm{d}_{6}$ acetone at $830{ }^{\circ} \mathrm{C}$ :<smiles>CC(=O)OC(C)=O</smiles>

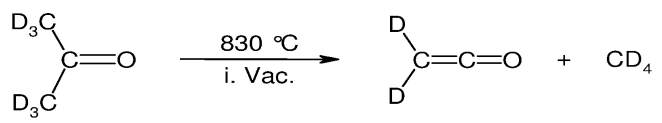

After intermediate trapping of the by-product acetic acid in one case and unreacted $\mathrm{d}_{6}$-acetone in the other case by a dry ice cold trap, ketene was collected in a trap held at liquid nitrogen temperature and purified through repeated distillations if necessary. Enough (4-D)ketene for a spectrum analysis was also obtained by the second reaction using partially deutereted acetone. 


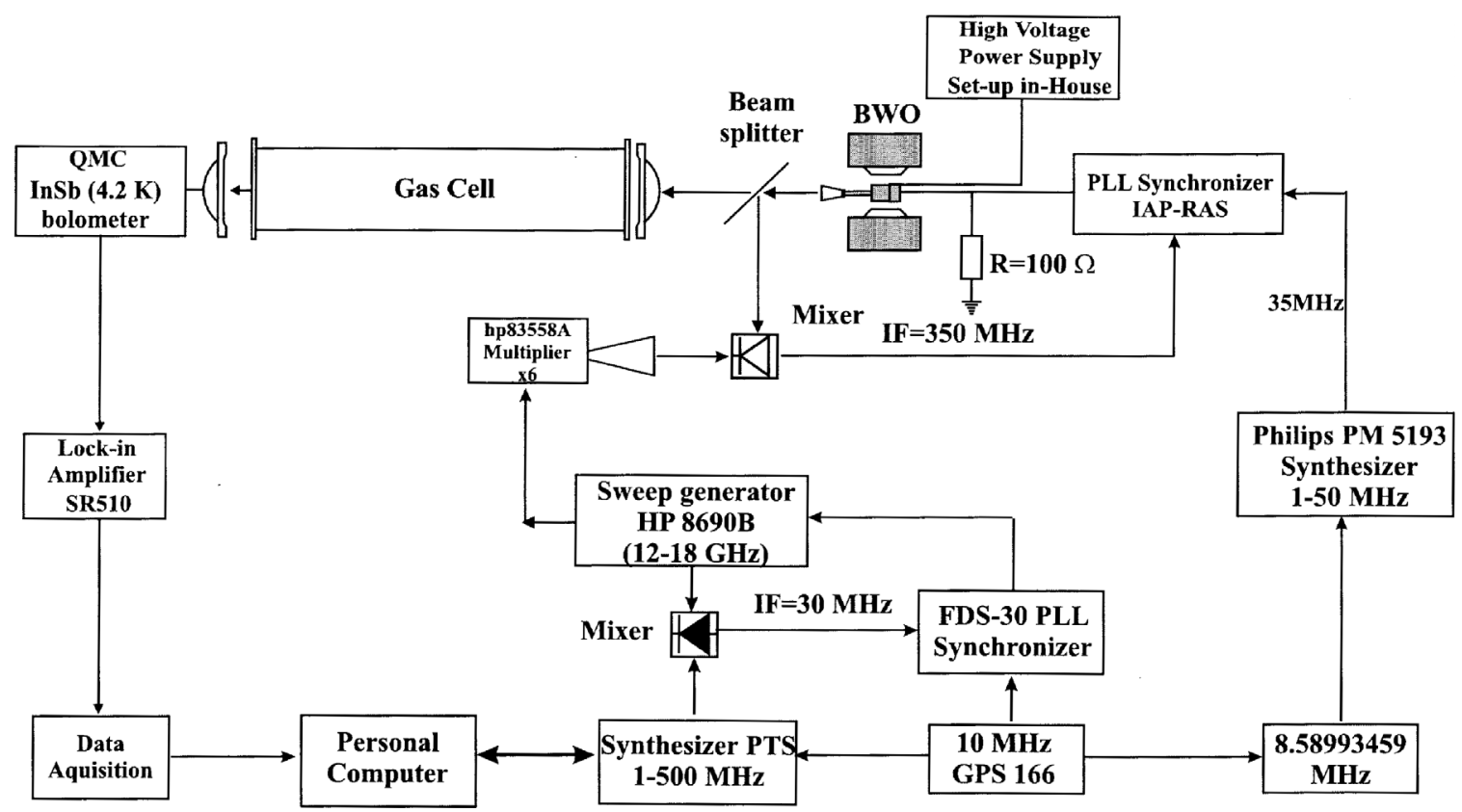

Fig. 1. Absorption submillimeterwave BWO-based spectrometer (Technische Fakultät der Universität Kiel). List of used devices: Istok BWO's OB 30, OB 32, OB 53; PLL Synchronizer - Inst. Appl. Physics, Russ. Acad. Sci., Nizhnii Novgorod; mixer with parabolic mirror - Inst. Appl. Physics, Russ. Acad. Sci., Nizhnii Novgorod; mixer $30 \mathrm{MHz}$ IF - prepared inhouse; sextupler hp 83558A; sweep Generator hp 8690B (12-18 GHz); Schomandl FDS 30 Synchronizer; synthesizer PTS 1-500 MHz; GPS 166 for $10 \mathrm{MHz}$ Standard; Converter $10 \mathrm{MHz}$ to $8.58993459 \mathrm{MHz}$ for driving the Philips PM 5193 Synthesizer; QMC InSb chip cooled down by a GM/JT refrigerator of Quantum Technology; Lock-in Amplifier Stanford Res. Instr. 510; data acquisition prepared in-house.

All measurements for the normal and the deuterated species have been performed without any ${ }^{13} \mathrm{C}$ and ${ }^{18} \mathrm{O}$ isotopic enrichment.

The millimeterwave spectra have been recorded using a PC-controlled spectrometer operating in the source modulation mode with Istok BWO's (backward wave oscillator) as direct radiation sources. The freespace absorption cell consisted of a 10 -cm-diameter glass tube, $4.20 \mathrm{~m}$ in length and capped at the ends with Teflon collimating lenses. Low-noise detection was achieved by use of an InSb bolometer cooled at 4.2 K by a cryocooler system of Quantum Technology (see Fig. 1).

Measurements in the frequency range $200-$ $350 \mathrm{GHz}$ were carried out at room temperature $\left(27{ }^{\circ} \mathrm{C}\right)$ at a pressure of about $1-3 \mathrm{~Pa}$. With respect to the weak intensity of the absorption lines and taking in account that several lines are partially overlapped by other strong lines of the ground state or excited vibrational states, the accuracy of the frequency measurements has been estimated to be $\pm 10 \mathrm{kHz}$ for strong lines and $\pm 20 \mathrm{kHz}$ for week unperturbed lines.

\section{Results of the Rotational Analysis}

3.1. $\mathrm{H}_{2}{ }^{13} \mathrm{C}={ }^{13} \mathrm{CO}$

Due to the weakness of the spectrum (abundance $0.02 \%$ ) the reported rotational lines of this isotopomer have been identified using the rotational constants calculated applying the mass dependence method of Watson et al. $[4,6,7]$ to the set of rotational constants of seven known isotopomers $[1,5]$.

The lines were found within intervals of $3-4 \mathrm{MHz}$ with respect to the frequencies obtained using the calculated constants.

$K_{a}=3$ doublets at high frequencies, moreover, were of great help for a correct identification of the lines. Other lines with odd $K_{a}$ values and finally those with even $K_{a}$ values - due to spin statistics the former are three times as strong as the latter - have been added. Through successive fits performed by use of Watson's S-reduced Hamiltonian $[8,9]$ in the $\mathrm{I}^{r}$ representation (with an asymmetry parameter of $\kappa=-0.997$ ketene is very near to the prolate top limit) several lines up to 
Table 1a. Selection of measured transition frequencies for the isotopomer $\mathrm{H}_{2}{ }^{13} \mathrm{C}^{13} \mathrm{CO}$.

\begin{tabular}{|c|c|c|c|c|c|c|c|c|}
\hline No. & $J$ & $K_{a}$ & $K_{c}$ & $J^{\prime}$ & $K_{a}{ }^{\prime}$ & $K_{c}^{\prime}$ & $f_{\text {obs }}(\mathrm{MHz})$ & $f_{\mathrm{o}-\mathrm{c}}(\mathrm{kHz})$ \\
\hline 1 & 11 & 0 & 11 & 10 & 0 & 10 & 215154.799 & -30 \\
\hline 2 & 11 & 1 & 10 & 10 & 1 & 9 & 217147.388 & 22 \\
\hline 3 & 11 & 3 & 8 & 10 & 3 & 7 & 215150.905 & 87 \\
\hline 4 & 11 & 5 & 6 & 10 & 5 & 5 & 214978.519 & -4 \\
\hline 5 & 11 & 7 & 4 & 10 & 7 & 3 & 214711.475 & 56 \\
\hline 6 & 12 & 0 & 12 & 11 & 0 & 11 & 234695.134 & 27 \\
\hline 7 & 12 & 1 & 11 & 11 & 1 & 10 & 236880.444 & -9 \\
\hline 8 & 12 & 1 & 12 & 11 & 1 & 11 & 232636.972 & -6 \\
\hline 9 & 12 & 2 & 10 & 11 & 2 & 9 & 234832.871 & -70 \\
\hline 10 & 12 & 2 & 11 & 11 & 2 & 10 & 234735.039 & -16 \\
\hline 11 & 12 & 3 & 10 & 11 & 3 & 9 & 234708.228 & 1 \\
\hline 12 & 12 & 3 & 9 & 11 & 3 & 8 & 234708.636 & -1 \\
\hline 13 & 12 & 4 & 8 & 11 & 4 & 7 & 234624.605 & 45 \\
\hline 14 & 13 & 2 & 12 & 12 & 2 & 11 & 254289.263 & 9 \\
\hline 15 & 13 & 3 & 10 & 12 & 3 & 9 & 254266.152 & 36 \\
\hline 16 & 13 & 3 & 11 & 12 & 3 & 10 & 254265.511 & 9 \\
\hline 17 & 13 & 4 & 9 & 12 & 4 & 8 & 254173.888 & 6 \\
\hline 18 & 13 & 5 & 8 & 12 & 5 & 7 & 254059.463 & 0 \\
\hline 19 & 14 & 0 & 14 & 13 & 0 & 13 & 273760.187 & -32 \\
\hline 20 & 14 & 1 & 13 & 13 & 1 & 12 & 276340.474 & -5 \\
\hline 21 & 15 & 4 & 11 & 14 & 4 & 10 & 293270.488 & -25 \\
\hline 22 & 15 & 5 & 10 & 14 & 5 & 9 & 293137.347 & -23 \\
\hline 23 & 15 & 7 & 8 & 14 & 7 & 7 & 292771.813 & 24 \\
\hline 24 & 16 & 0 & 16 & 15 & 0 & 15 & 312802.030 & 23 \\
\hline 25 & 16 & 1 & 15 & 15 & 1 & 14 & 315791.170 & 10 \\
\hline 26 & 16 & 1 & 16 & 15 & 1 & 15 & 310136.293 & -19 \\
\hline 27 & 16 & 2 & 14 & 15 & 2 & 13 & 313173.658 & 45 \\
\hline 28 & 16 & 3 & 14 & 15 & 3 & 13 & 312934.559 & 7 \\
\hline 29 & 17 & 1 & 16 & 16 & 1 & 15 & 335512.526 & -35 \\
\hline 30 & 17 & 2 & 15 & 16 & 2 & 14 & 332766.622 & 11 \\
\hline
\end{tabular}

The standard deviation of the complete least squares fit of 86 transition frequencies is $30 \mathrm{kHz}$. A complete list of all measured transition frequencies is available under the number TNA 41 at the central library of the University of Kiel or at the Sektion für Spektren und Strukturdokumentation, University of Ulm (Germany).

$K_{a}=7$ could be identified. Like in the case of the papers $[1,5,10]$, increasing deviations from the predicted frequencies occurred for higher $K_{a}$ values. Thus only lines up to $K_{a, \max }=7$ were included in the fit. Table 1a gives a selection of measured transitions, and Table $1 b$ reports the obtained parameters with the corresponding correlation matrix of the least squares fit. A complete list of all measured transition frequencies is deposited under the number TNA 41 at the central library of the University of Kiel. It is also available upon request from the Sektion für Spektren und Strukturdokumentation, University of Ulm (Germany).

The reported molecular parameters of Table $1 \mathrm{~b}$ are considered to give a reliable description of the rotational spectrum in the vibrational ground state of $\mathrm{H}_{2}{ }^{13} \mathrm{C}={ }^{13} \mathrm{CO}$.

Because of the $C_{2 v}$ symmetry of the molecule, only a-type transitions, $\Delta K_{a}=0$, could be observed. Thus

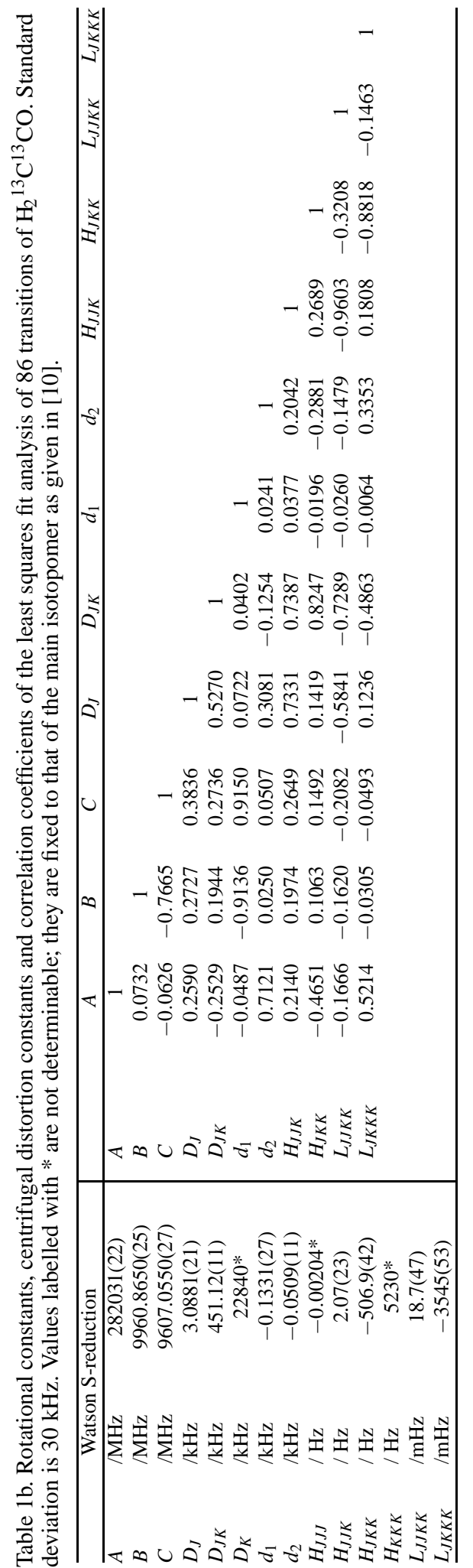


Table 1c. Constants of the $\mathrm{H}_{2}{ }^{13} \mathrm{C}^{13} \mathrm{CO}$ isotopomer compared with ${ }^{13} \mathrm{C}$ monosubstituted isotopomers.

\begin{tabular}{lcccc}
\hline Parameter & & $\mathrm{H}_{2}{ }^{13} \mathrm{C}^{13} \mathrm{CO}$ & $\mathrm{H}_{2} \mathrm{C}^{13} \mathrm{CO}^{\mathrm{a}}$ & $\mathrm{H}_{2}{ }^{13} \mathrm{CCO}^{\mathrm{a}}$ \\
\hline$A$ & $/ \mathrm{MHz}$ & $282031(22)$ & $282037(26)$ & $282044(14)$ \\
$B$ & $/ \mathrm{MHz}$ & $9960.8650(25)$ & $10293.6281(11)$ & $9960.9799(6)$ \\
$C$ & $/ \mathrm{MHz}$ & $9607.0550(27)$ & $9916.2102(11)$ & $9607.1413(6)$ \\
$D_{J}$ & $/ \mathrm{kHz}$ & $3.0881(21)$ & $3.272(2)$ & $3.0903(12)$ \\
$D_{J K}$ & $/ \mathrm{kHz}$ & $451.12(11)$ & $477.53(14)$ & $452.11(8)$ \\
$D_{K}$ & $/ \mathrm{kHz}$ & $22840^{*}$ & $22840^{*}$ & $22840^{*}$ \\
$d_{1}$ & $/ \mathrm{kHz}$ & $-0.1331(27)$ & $-0.1467(14)$ & $-0.13297(57)$ \\
$d_{2}$ & $/ \mathrm{kHz}$ & $-0.0509(11)$ & $-0.0589(14)$ & $-0.04872(57)$ \\
$H_{J J J}$ & $/ \mathrm{Hz}$ & $-0.00204^{*}$ & $-0.00204^{*}$ & $-0.00204^{*}$ \\
$H_{J J K}$ & $/ \mathrm{Hz}$ & $2.07(23)$ & $1.77(25)$ & $2.29(14)$ \\
$H_{K K J}$ & $/ \mathrm{Hz}$ & $-506.9(42)$ & $-537.4(52)$ & $-489.1(28)$ \\
$H_{K K K}$ & $/ \mathrm{Hz}$ & $5230^{*}$ & $5230^{*}$ & $5230^{*}$ \\
$L_{J J K K}$ & $/ \mathrm{mHz}$ & $18.7(47)$ & $29.2(53)$ & $14.0(28)$ \\
$L_{J K K K}$ & $/ \mathrm{mHz}$ & $-3545(53)$ & $-3930(60)$ & $-3224(34)$ \\
$\sigma_{\text {Fit }}$ & $/ \mathrm{kHz}$ & 30 & 46 & 25 \\
No. of lines fitted & 86 & 141 & 132 \\
\hline
\end{tabular}

* Not determinable. Value fixed to that of the main isotopomer $\mathrm{H}_{2} \mathrm{CCO}$ as given in [10]. ${ }^{\mathrm{a}}$ Data of [1].

the purely $K$-dependent centrifugal distortion constants $D_{K}$ and $H_{K K K}$ were fixed to the corresponding $\mathrm{H}_{2} \mathrm{C}=\mathrm{CO}$ values [10]. The value of the $D_{K}$ constant has not been adjusted in dependence of the mass change of the isotopomers because this change would have been within the given uncertainty. Together with $H_{K K K}$, also the very small $H_{J J J}$ centrifugal distortion constant has been held fixed to the value of [10]; further two octic constants, $L_{J J K K}$ and $L_{J K K K}$, were included in the fit, as it has been done for all isotopomers [1,5]. The value obtained for the rotational constant $A$ is comparable with the values obtained for the main and other isotopomers $[1,5]$.

For the presently investigated isotopomer, the least squares fit has been carried out using 86 transitions of the type $\Delta J=1, \Delta K_{a}=0$ with a resulting standard deviation of $30 \mathrm{kHz}$. A few lines with a least squares fit deviation larger than one standard deviation but smaller than three standard deviations have been nevertheless included in the complete list: their frequency measurements were influenced by the closeness of other strong lines.

In Table 1c the resulting data are reported in comparison with those of the monosubstituted ${ }^{13} \mathrm{C}$ isotopomers of investigation [1].

$$
\text { 3.2. } \mathrm{D}_{2}{ }^{13} \mathrm{C}=\mathrm{CO}, \mathrm{D}_{2} \mathrm{C}={ }^{13} \mathrm{CO} \text { and } \mathrm{D}_{2} \mathrm{C}=\mathrm{C}^{18} \mathrm{O}
$$

The reported rotational lines of these three isotopomers have been first calculated with rotational constants obtained by the $r_{\mathrm{m}}$-program, as in the preced- ing case, but using now the set of 8 known isotopomers and adding then each time the constants of the newly assigned one. The natural abundance of these species in the prepared $\mathrm{D}_{2} \mathrm{C}=\mathrm{CO}$ is $1 \%$ for both ${ }^{13} \mathrm{C}$ isotopomers and $0.2 \%$ for the ${ }^{18} \mathrm{O}$ isotopomer. The differences between observed and predicted values of the transition frequencies were in the range of about 1 to $3 \mathrm{MHz}$, thus confirming the reliability of the $\mathrm{r}_{\mathrm{m}}$-parameters for a prediction of the spectrum of isotopically substituted species with low abundance [4].

Like in (4,5-D)ketene [11] it is possible, by these isotopomers, to consider in the fit also lines with $\mathrm{K}_{a}=$ 8 or 9 without having deviations due to coupling with low excited vibrational states through a centrifugal distortion resonance with high $K_{a}$ [12]. Due to the $\mathrm{C}_{2 v}$ symmetry of the molecule and the measurements of only $a$-type lines, $\Delta K_{a}=0$, purely $K$-dependent centrifugal distortion constants $D_{K}$ and $H_{K K K}$ could not be determined and were fixed to the value of $\Delta_{K}$ and $H_{K K K}$ published in [13], knowing that the difference between $D_{K}$ and $\Delta_{K}$ is negligible [14]. Also for $H_{J J J}$ the $a b$ initio value given in [3] for $\mathrm{D}_{2} \mathrm{C}=\mathrm{CO}$ has been used. For each of these species a set of more than 100 transitions for each isotopomer in the range $200-340 \mathrm{GHz}$ has been employed for the least squares analysis.

Tables 2a, 3a and 4a report a selection of measured transition frequencies while the complete lists can be requested from the central library of the University of Kiel under the number TNA 41 or at the Sektion für Spektren und Strukturdokumentation, University of Ulm (Germany).

Tables $2 b, 3 b$ and $4 b$ report the results of the least squares fit with the corresponding standard deviations and correlation matrices.

\section{3. $\mathrm{D}_{2} \mathrm{C}=\mathrm{CO}$ and $\mathrm{DHC}=\mathrm{CO}$}

The spectra of these two isotopomers have been investigated and measured several years ago in the millimeterwave range by $\mathrm{L}$. Nemes and M. Winnewisser [11]. In addition to several Q-branch frequencies $\left(\Delta J=0, \Delta K_{a}=0\right)$, they reported R-branch $\left(\Delta J=1, \Delta K_{a}=0\right)$ transition frequencies up to $J=10$, but because all the presently investigated isotopomers have been measured up to higher $J$-values and higher frequencies, the spectra of these two isotopomers have been supplemented with higher $J$-values in order to have comparable spectral informations for the least squares fit. 
Table 2a. Selection of measured transition frequencies for the isotopomer $\mathrm{D}_{2}{ }^{13} \mathrm{CCO}$.

\begin{tabular}{rrrrrrrrr}
\hline No. & $J$ & $K_{a}$ & $K_{c}$ & $J^{\prime}$ & $K_{a}{ }^{\prime}$ & $K_{c}{ }^{\prime}$ & $f_{\mathrm{obs}}(\mathrm{MHz})$ & $f_{\mathrm{o}-\mathrm{c}}(\mathrm{kHz})$ \\
\hline 1 & 11 & 2 & 10 & 10 & 2 & 9 & 189547.654 & -10 \\
2 & 11 & 2 & 9 & 10 & 2 & 8 & 189908.343 & -5 \\
3 & 11 & 3 & 9 & 10 & 3 & 8 & 189616.132 & 1 \\
4 & 11 & 3 & 8 & 10 & 3 & 7 & 189620.133 & -1 \\
5 & 11 & 4 & 8 & 10 & 4 & 7 & 189548.618 & 18 \\
6 & 12 & 2 & 11 & 11 & 2 & 10 & 206764.129 & -5 \\
7 & 12 & 3 & 9 & 11 & 3 & 8 & 206866.166 & 8 \\
8 & 12 & 3 & 10 & 11 & 3 & 9 & 206859.930 & -3 \\
9 & 12 & 4 & 8 & 11 & 4 & 7 & 206782.776 & -21 \\
10 & 13 & 0 & 13 & 12 & 0 & 12 & 223506.642 & -2 \\
11 & 13 & 1 & 12 & 12 & 1 & 11 & 227455.443 & -14 \\
12 & 13 & 1 & 13 & 12 & 1 & 12 & 220440.099 & 15 \\
13 & 13 & 2 & 12 & 12 & 2 & 11 & 223976.646 & -13 \\
14 & 13 & 3 & 10 & 12 & 3 & 9 & 224114.425 & -5 \\
15 & 13 & 3 & 11 & 12 & 3 & 10 & 224105.089 & -5 \\
16 & 13 & 4 & 10 & 12 & 4 & 9 & 224017.587 & 14 \\
17 & 14 & 4 & 11 & 13 & 4 & 10 & 241253.117 & 43 \\
18 & 14 & 5 & 10 & 13 & 5 & 9 & 241155.001 & -4 \\
19 & 14 & 5 & 9 & 13 & 5 & 8 & 241155.001 & -4 \\
20 & 14 & 6 & 9 & 13 & 6 & 8 & 241047.450 & -5 \\
21 & 14 & 6 & 8 & 13 & 6 & 7 & 241047.450 & -5 \\
22 & 14 & 7 & 8 & 13 & 7 & 7 & 240924.996 & 2 \\
23 & 14 & 7 & 7 & 13 & 7 & 6 & 240924.996 & 2 \\
24 & 18 & 3 & 16 & 17 & 3 & 15 & 310351.765 & -6 \\
25 & 18 & 3 & 15 & 17 & 3 & 14 & 310399.854 & 8 \\
26 & 18 & 4 & 15 & 17 & 4 & 14 & 310203.019 & -1 \\
27 & 18 & 4 & 14 & 17 & 4 & 13 & 310203.581 & -2 \\
28 & 19 & 2 & 18 & 18 & 2 & 17 & 327150.714 & -10 \\
29 & 19 & 2 & 17 & 18 & 2 & 16 & 328971.763 & 26 \\
30 & 19 & 3 & 17 & 18 & 3 & 16 & 327605.08 & -12 \\
31 & 19 & 3 & 16 & 18 & 3 & 15 & 327668.129 & -6 \\
32 & 19 & 7 & 13 & 18 & 7 & 12 & 326959.123 & -12 \\
33 & 19 & 8 & 12 & 18 & 8 & 11 & 326765.275 & 0 \\
\hline & & & & & & & & \\
& \\
1 & & & \\
13 &
\end{tabular}

The standard deviation of the complete least squares fit of 131 transition frequencies is $22 \mathrm{kHz}$. A complete list of all measured transition frequencies is available under the number TNA 41 at the central library of the University of Kiel or at the Sektion für Spektren und Strukturdokumentation, University of Ulm (Germany).

Tables 5a and 6a give a selection of the transitions measured for extending the frequency range of these two isotopomers while the complete list of lines including those measured in [11], are deposited in the central library of the University of Kiel under the number TNA 41 and can be requested as in the preceding cases.

The fit of all the available transition frequencies has been performed using the Watson S-reduction centrifugal distortion program $[8,9]$ up to the 8 th order. Also in the case of $\mathrm{D}_{2} \mathrm{C}=\mathrm{CO}$ the same values for $D_{K}, H_{K K K}$, and $H_{J J J}$ have been used as it was made for the preceding considered perdeuterated isotopomers. In the case of $\mathrm{DHC}=\mathrm{CO}$ the value of $D_{K}$ has been taken from [15], and the values for $H_{K K K}$ and $H_{J J J}$ were fixed

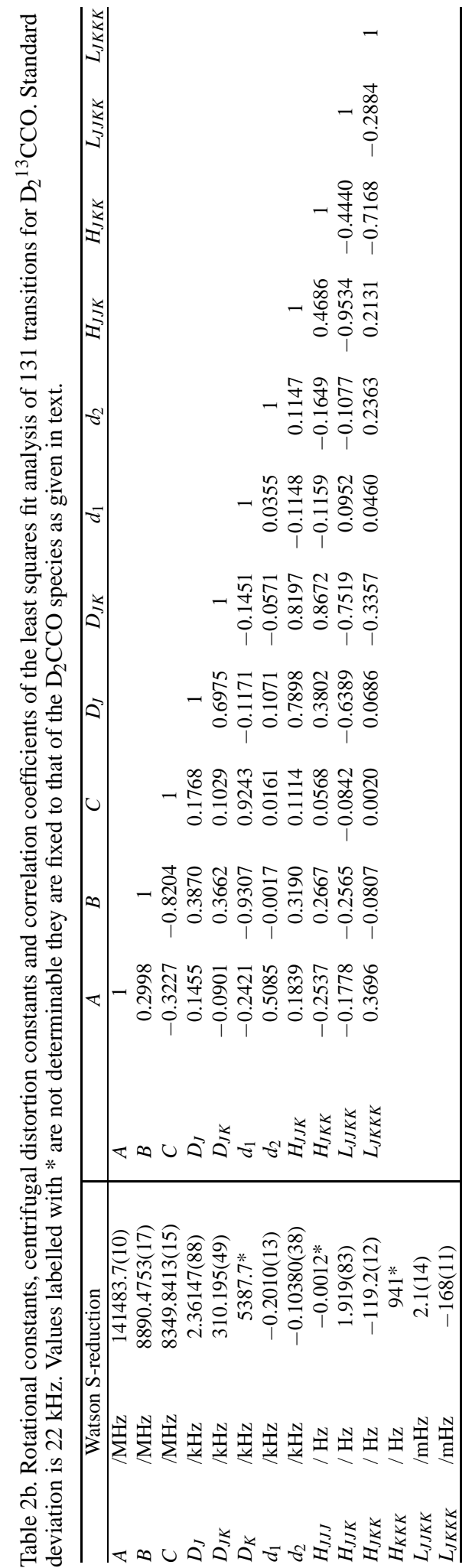


Table 3a. Selection of measured transition frequencies for the isotopomer $\mathrm{D}_{2} \mathrm{C}^{13} \mathrm{CO}$.

\begin{tabular}{rrrrrrrrr}
\hline No. & \multicolumn{1}{c}{$J$} & $K_{a}$ & $K_{c}$ & $J^{\prime}$ & $K_{a}{ }^{\prime}$ & $K_{c}{ }^{\prime}$ & $f_{\text {obs }}(\mathrm{MHz})$ & $f_{\mathrm{o}-\mathrm{c}}(\mathrm{kHz})$ \\
\hline 1 & 12 & 0 & 12 & 11 & 0 & 11 & 211514.844 & -1 \\
2 & 12 & 1 & 11 & 11 & 1 & 10 & 215300.818 & -2 \\
3 & 12 & 2 & 11 & 11 & 2 & 10 & 211921.499 & -10 \\
4 & 12 & 3 & 9 & 11 & 3 & 8 & 212036.956 & -5 \\
5 & 12 & 3 & 10 & 11 & 3 & 9 & 212029.736 & 15 \\
6 & 12 & 4 & 9 & 11 & 4 & 8 & 211948.122 & 24 \\
7 & 12 & 5 & 8 & 11 & 5 & 7 & 211864.354 & -8 \\
8 & 12 & 6 & 7 & 11 & 6 & 6 & 211770.399 & -10 \\
9 & 12 & 7 & 6 & 11 & 7 & 5 & 211662.300 & -4 \\
10 & 12 & 8 & 4 & 11 & 8 & 3 & 211538.004 & -27 \\
11 & 13 & 0 & 13 & 12 & 0 & 12 & 229040.847 & -17 \\
12 & 13 & 1 & 13 & 12 & 1 & 12 & 225844.302 & 15 \\
13 & 13 & 1 & 12 & 12 & 1 & 11 & 233212.832 & 23 \\
14 & 13 & 2 & 12 & 12 & 2 & 11 & 229562.086 & -12 \\
15 & 13 & 2 & 11 & 12 & 2 & 10 & 230219.146 & -26 \\
16 & 13 & 3 & 11 & 12 & 3 & 10 & 229706.473 & -31 \\
17 & 13 & 3 & 10 & 12 & 3 & 9 & 229717.355 & -6 \\
18 & 15 & 0 & 15 & 14 & 0 & 14 & 264021.587 & 7 \\
19 & 15 & 1 & 15 & 14 & 1 & 14 & 260522.940 & -4 \\
20 & 15 & 1 & 14 & 14 & 1 & 13 & 269013.766 & -8 \\
21 & 15 & 2 & 14 & 14 & 2 & 13 & 264828.863 & -4 \\
22 & 15 & 2 & 13 & 14 & 2 & 12 & 265834.140 & 9 \\
23 & 15 & 3 & 13 & 14 & 3 & 12 & 265064.735 & 16 \\
24 & 15 & 3 & 12 & 14 & 3 & 11 & 265087.090 & 16 \\
25 & 17 & 0 & 17 & 16 & 0 & 16 & 298898.779 & -20 \\
26 & 17 & 1 & 17 & 16 & 1 & 16 & 295174.942 & -6 \\
27 & 17 & 1 & 16 & 16 & 1 & 15 & 304779.321 & -8 \\
28 & 17 & 2 & 16 & 16 & 2 & 15 & 300074.056 & 4 \\
29 & 17 & 2 & 15 & 16 & 2 & 14 & 301526.037 & -3 \\
30 & 18 & 0 & 18 & 17 & 0 & 17 & 316296.122 & 4 \\
31 & 18 & 1 & 18 & 17 & 1 & 17 & 312490.189 & -8 \\
32 & 18 & 1 & 17 & 17 & 1 & 16 & 322646.963 & 12 \\
33 & 18 & 2 & 16 & 17 & 2 & 15 & 319403.896 & -35 \\
34 & 19 & 0 & 19 & 18 & 0 & 18 & 333665.025 & 7 \\
\hline
\end{tabular}

The standard deviation of the complete least squares fit on 102 transition frequencies is $33 \mathrm{kHz}$. A complete list of all measured transition frequencies is available under the number TNA 41 at the central library of the University of Kiel or at the Sektion für Spektren und Strukturdokumentation, University of Ulm (Germany).

to the mean values of the two isotopomers $\mathrm{H}_{2} \mathrm{C}=\mathrm{CO}$ and $\mathrm{D}_{2} \mathrm{C}=\mathrm{CO}$.

The results of the least squares fit procedure are reported in Tables $5 \mathrm{~b}$ and $6 \mathrm{~b}$, together with the corresponding correlation matrix, the standard deviation and the number of transitions used.

\section{Conclusions}

Table 7 gives the values of the rotational constants together with the inertial moments and the inertia defect for all the ketene isotopomers so far investigated.

The data reported for the mother species, written in italics, are only given for comparison because they re-

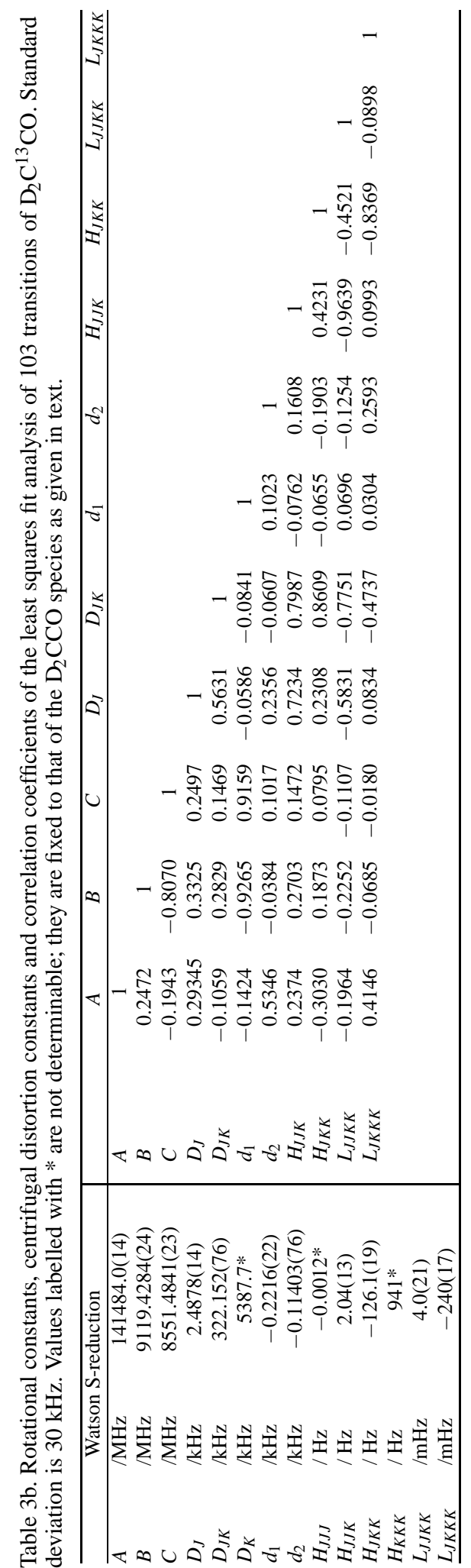


Table 4a. Selection of measured transitions frequencies for the isotopomer $\mathrm{D}_{2} \mathrm{CC}^{18} \mathrm{O}$.

\begin{tabular}{|c|c|c|c|c|c|c|c|c|}
\hline No. & $J$ & $K_{a}$ & $K_{c}$ & $J^{\prime}$ & $K_{a}^{\prime}$ & $K_{c}^{\prime}$ & $f_{\text {obs }}(\mathrm{MHz})$ & $f_{\mathrm{o}-\mathrm{c}}(\mathrm{kHz})$ \\
\hline 1 & 12 & 2 & 10 & 11 & 2 & 9 & 201572.257 & 13 \\
\hline 2 & 12 & 3 & 9 & 11 & 3 & 8 & 201242.297 & 31 \\
\hline 3 & 12 & 3 & 10 & 11 & 3 & 9 & 201237.029 & 25 \\
\hline 4 & 13 & 0 & 13 & 12 & 0 & 12 & 217480.836 & -13 \\
\hline 5 & 13 & 1 & 12 & 12 & 1 & 11 & 221196.386 & -10 \\
\hline 6 & 13 & 2 & 11 & 12 & 2 & 10 & 218432.321 & -6 \\
\hline 7 & 14 & 1 & 14 & 13 & 1 & 13 & 231033.635 & -5 \\
\hline 8 & 14 & 1 & 13 & 13 & 1 & 12 & 238183.075 & -6 \\
\hline 9 & 14 & 2 & 12 & 13 & 2 & 11 & 235306.879 & -15 \\
\hline 10 & 14 & 2 & 13 & 13 & 2 & 12 & 234642.69 & -2 \\
\hline 11 & 14 & 3 & 12 & 13 & 3 & 11 & 234789.769 & 4 \\
\hline 12 & 14 & 3 & 11 & 13 & 3 & 10 & 234801.256 & 18 \\
\hline 13 & 14 & 0 & 14 & 13 & 0 & 13 & 234115.630 & -14 \\
\hline 14 & 15 & 0 & 15 & 14 & 0 & 14 & 250730.117 & -11 \\
\hline 15 & 15 & 1 & 14 & 14 & 1 & 13 & 255163.166 & -13 \\
\hline 16 & 15 & 2 & 14 & 14 & 2 & 13 & 251381.346 & 3 \\
\hline 17 & 15 & 2 & 13 & 14 & 2 & 12 & 252196.795 & 12 \\
\hline 18 & 15 & 3 & 13 & 14 & 3 & 12 & 251567.968 & -44 \\
\hline 19 & 15 & 3 & 12 & 14 & 3 & 11 & 251584.227 & -33 \\
\hline 20 & 16 & 0 & 16 & 15 & 0 & 15 & 267323.201 & -9 \\
\hline 21 & 16 & 1 & 16 & 15 & 1 & 15 & 263976.159 & 5 \\
\hline 22 & 16 & 1 & 15 & 15 & 1 & 14 & 272136.138 & 17 \\
\hline 23 & 16 & 2 & 15 & 15 & 2 & 14 & 268115.530 & -15 \\
\hline 24 & 16 & 2 & 14 & 15 & 2 & 13 & 269102.754 & 26 \\
\hline 25 & 16 & 3 & 14 & 15 & 3 & 13 & 268347.522 & 15 \\
\hline 26 & 17 & 4 & 14 & 16 & 4 & 13 & 285002.137 & 8 \\
\hline 27 & 17 & 4 & 13 & 16 & 4 & 12 & 285002.431 & 2 \\
\hline 28 & 17 & 5 & 13 & 16 & 5 & 12 & 284880.721 & -43 \\
\hline 29 & 17 & 6 & 12 & 16 & 6 & 11 & 284752.389 & 3 \\
\hline 30 & 17 & 7 & 11 & 16 & 7 & 10 & 284608.562 & -6 \\
\hline 31 & 17 & 8 & 10 & 16 & 8 & 9 & 284445.369 & 8 \\
\hline 32 & 20 & 0 & 20 & 19 & 0 & 19 & 333464.293 & -7 \\
\hline 33 & 20 & 1 & 20 & 19 & 1 & 19 & 329789.429 & 4 \\
\hline 34 & 20 & 2 & 19 & 19 & 2 & 18 & 335002.010 & -6 \\
\hline 35 & 20 & 2 & 18 & 19 & 2 & 17 & 336897.100 & 64 \\
\hline
\end{tabular}

The standard deviation of the complete least squares fit of 115 transition frequencies is $29 \mathrm{kHz}$. A complete list of all measured transition frequencies is available under the number TNA 41 at the central library of the University Kiel or at the Sektion für Spektren und Strukturdokumentation, University of Ulm (Germany).

sult from an IR + MW combined fit [10]. All other data are the result of a pure rotational spectrum investigation.

It can be seen that the values for the constant $A$ of the six hydrogenated isotopic species are now all contained in a range of $36 \mathrm{MHz}$ with a mean value of $282,036 \mathrm{MHz}$ and therefore practically of a similar value for all the investigated isotopomers. The determination uncertainty is due to measurements of the only allowed $\mu_{a}$-spectrum and to the changed mass of the substituted isotopomer. This fact is also apparent for the four perdeutereted isotopomers with $C_{2 v}$ symmetry, where the variability range reduces to $8 \mathrm{MHz}$ in agreement with the smaller oscillation amplitude of

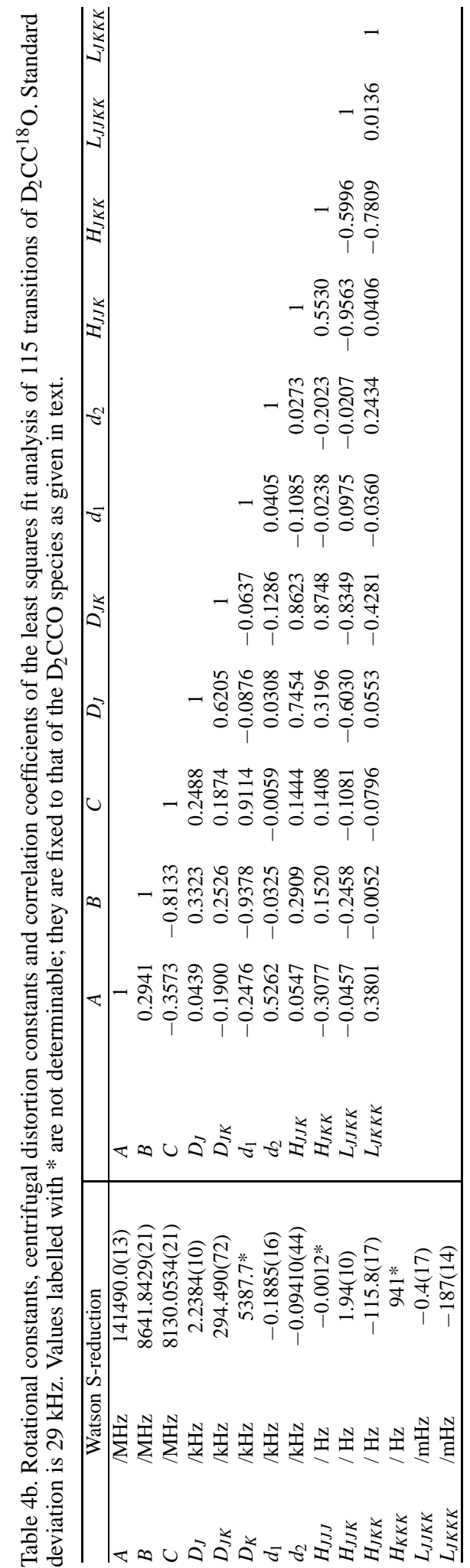


Table 5a. Selection of transition frequencies of the isotopomer $\mathrm{D}_{2} \mathrm{CCO}$ measured in this investigation.

\begin{tabular}{rrrrrrrrr}
\hline No. & $J$ & $K_{a}$ & $K_{c}$ & $J^{\prime}$ & $K_{a}{ }^{\prime}$ & $K_{c}{ }^{\prime}$ & $f_{\text {obs }}(\mathrm{MHz})$ & $f_{\mathrm{o}-\mathrm{c}}(\mathrm{kHz})$ \\
\hline 1 & 12 & 0 & 12 & 11 & 0 & 11 & 211545.992 & 29 \\
2 & 12 & 1 & 11 & 11 & 1 & 10 & 215333.278 & -16 \\
3 & 12 & 2 & 10 & 11 & 2 & 9 & 212470.447 & -6 \\
4 & 12 & 2 & 11 & 11 & 2 & 10 & 211952.834 & 14 \\
5 & 13 & 0 & 13 & 12 & 0 & 12 & 229074.506 & -9 \\
6 & 13 & 2 & 12 & 12 & 2 & 11 & 229596.001 & -6 \\
7 & 13 & 2 & 11 & 12 & 2 & 10 & 230253.499 & 1 \\
8 & 13 & 3 & 11 & 12 & 3 & 10 & 229740.433 & 3 \\
9 & 13 & 3 & 10 & 12 & 3 & 9 & 229751.301 & 3 \\
10 & 13 & 4 & 10 & 12 & 4 & 9 & 229647.615 & 31 \\
11 & 13 & 4 & 9 & 12 & 4 & 8 & 229647.615 & -37 \\
12 & 13 & 6 & 8 & 12 & 6 & 7 & 229451.060 & -1 \\
13 & 13 & 7 & 7 & 12 & 7 & 6 & 229333.080 & -13 \\
14 & 13 & 8 & 6 & 12 & 8 & 5 & 229197.972 & -8 \\
15 & 13 & 9 & 5 & 12 & 9 & 4 & 229044.108 & 9 \\
16 & 15 & 0 & 15 & 14 & 0 & 14 & 264060.244 & -13 \\
17 & 15 & 1 & 15 & 14 & 1 & 14 & 260560.623 & -22 \\
18 & 15 & 1 & 14 & 14 & 1 & 13 & 269054.296 & -28 \\
19 & 15 & 2 & 14 & 14 & 2 & 13 & 264867.982 & 15 \\
20 & 15 & 2 & 13 & 14 & 2 & 12 & 265873.866 & -3 \\
21 & 15 & 3 & 13 & 14 & 3 & 12 & 265103.891 & 8 \\
22 & 15 & 3 & 12 & 14 & 3 & 11 & 265126.274 & 15 \\
23 & 15 & 4 & 12 & 14 & 4 & 11 & 264986.811 & 117 \\
24 & 15 & 4 & 11 & 14 & 4 & 10 & 264986.811 & -72 \\
25 & 19 & 0 & 19 & 18 & 0 & 18 & 333713.550 & -5 \\
26 & 19 & 1 & 19 & 18 & 1 & 18 & 329845.572 & 25 \\
27 & 19 & 1 & 18 & 18 & 1 & 17 & 340554.631 & -23 \\
28 & 19 & 2 & 18 & 18 & 2 & 17 & 335344.258 & -13 \\
29 & 19 & 2 & 17 & 18 & 2 & 16 & 337352.344 & -1 \\
30 & 19 & 3 & 17 & 18 & 3 & 16 & 335848.900 & 4 \\
31 & 19 & 3 & 16 & 18 & 3 & 15 & 335922.273 & 10 \\
32 & 19 & 4 & 16 & 18 & 4 & 15 & 335676.406 & -11 \\
33 & 19 & 4 & 15 & 18 & 4 & 14 & 335677.458 & 30 \\
\hline
\end{tabular}

The standard deviation of the complete least squares fit of 183 transition frequencies is $27 \mathrm{kHz}$. A complete list of all measured transition frequencies is available under the number TNA 41 at the central library of the University of Kiel or at the Sektion für Spektren und Strukturdokumentation, University of Ulm (Germany).

deuterium. The derived rotational constants given in Table 7 represent now, with the new investigated isotopomers, a group of eleven sets of data, which will be the basis for a mass-dependent structure calculation after Watson et al. [4] in a following paper. Due to the definition of an $r_{m}$-structure, a good agreement with the $a b$ initio $\mathrm{r}_{\mathrm{e}}$-structure of paper [3] is now to be expected, reflecting the increased number of available rotational constants.

\section{Aknowledgements}

I wish to thank Prof. R. Knoechel and the Technical Faculty of the University of Kiel for research facilities. My gratitude goes also to the central workshop of

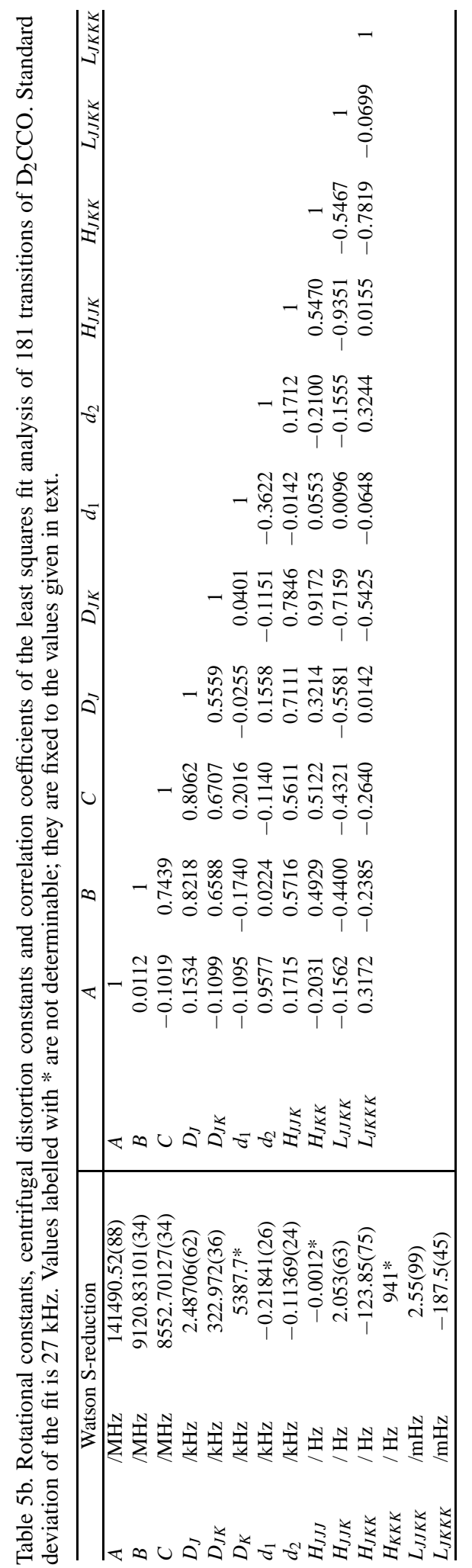


Table 6a. Selection of transition frequencies of the isotopomer DHCCO measured in this investigation.

\begin{tabular}{|c|c|c|c|c|c|c|c|c|}
\hline No. & $J$ & $K_{a}$ & $K_{c}$ & $J^{\prime}$ & $K_{a}^{\prime}$ & $K_{c}^{\prime}$ & $f_{\text {obs }}(\mathrm{MHz})$ & $f_{\mathrm{o}-\mathrm{c}}(\mathrm{kHz}$ \\
\hline 1 & 12 & 0 & 12 & 11 & 0 & 11 & 225581.294 & -1 \\
\hline 2 & 12 & 1 & 12 & 11 & 1 & 11 & 222936.522 & -24 \\
\hline 3 & 12 & 1 & 11 & 11 & 1 & 10 & 228600.345 & -7 \\
\hline 4 & 12 & 2 & 11 & 11 & 2 & 10 & 225767.935 & -11 \\
\hline 5 & 12 & 2 & 10 & 11 & 2 & 9 & 226025.242 & 12 \\
\hline 6 & 13 & 1 & 13 & 12 & 1 & 12 & 241499.152 & -4 \\
\hline 7 & 13 & 1 & 12 & 12 & 1 & 11 & 247633.465 & -8 \\
\hline 8 & 13 & 2 & 12 & 12 & 2 & 11 & 244570.010 & 38 \\
\hline 9 & 13 & 2 & 11 & 12 & 2 & 10 & 244897.165 & -2 \\
\hline 10 & 13 & 3 & 11 & 12 & 3 & 10 & 244620.361 & 23 \\
\hline 11 & 13 & 3 & 10 & 12 & 3 & 9 & 244623.539 & -1 \\
\hline 12 & 13 & 4 & 10 & 12 & 4 & 9 & 244541.841 & 24 \\
\hline 13 & 15 & 0 & 15 & 14 & 0 & 14 & 281780.467 & -6 \\
\hline 14 & 15 & 1 & 15 & 14 & 1 & 14 & 278613.289 & 7 \\
\hline 15 & 15 & 1 & 14 & 14 & 1 & 13 & 285686.932 & 3 \\
\hline 16 & 15 & 2 & 14 & 14 & 2 & 13 & 282165.200 & 9 \\
\hline 17 & 15 & 2 & 13 & 14 & 2 & 12 & 282667.453 & -29 \\
\hline 18 & 15 & 3 & 13 & 14 & 3 & 12 & 282258.556 & -6 \\
\hline 19 & 15 & 3 & 12 & 14 & 3 & 11 & 282265.153 & -3 \\
\hline 20 & 15 & 4 & 12 & 14 & 4 & 11 & 282162.067 & 11 \\
\hline 21 & 15 & 4 & 11 & 14 & 4 & 10 & 282162.067 & -22 \\
\hline 22 & 15 & 5 & 11 & 14 & 5 & 10 & 282058.120 & 7 \\
\hline 23 & 15 & 6 & 10 & 14 & 6 & 9 & 281938.087 & -22 \\
\hline 24 & 15 & 7 & 9 & 14 & 7 & 8 & 281797.484 & 19 \\
\hline 25 & 17 & 0 & 17 & 16 & 0 & 16 & 319177.003 & 14 \\
\hline 26 & 17 & 1 & 17 & 16 & 1 & 16 & 315711.039 & 21 \\
\hline 27 & 17 & 1 & 16 & 16 & 1 & 15 & 323720.925 & -25 \\
\hline 28 & 17 & 2 & 16 & 16 & 2 & 15 & 319747.179 & 7 \\
\hline 29 & 17 & 2 & 15 & 16 & 2 & 14 & 320476.799 & 18 \\
\hline 30 & 17 & 3 & 15 & 16 & 3 & 14 & 319898.065 & -3 \\
\hline 31 & 17 & 3 & 14 & 16 & 3 & 13 & 319910.455 & 4 \\
\hline 32 & 17 & 4 & 14 & 16 & 4 & 13 & 319781.615 & 11 \\
\hline 33 & 17 & 4 & 13 & 16 & 4 & 12 & 319781.615 & -70 \\
\hline 34 & 17 & 5 & 13 & 16 & 5 & 12 & 319659.820 & 5 \\
\hline 35 & 17 & 6 & 12 & 16 & 6 & 11 & 319521.789 & 9 \\
\hline 36 & 17 & 7 & 11 & 16 & 7 & 10 & 319361.288 & 27 \\
\hline
\end{tabular}

The standard deviation of the complete least squares fit of 159 transition frequencies is $23 \mathrm{kHz}$. A complete list of all measured transition frequencies is available under the number TNA 41 at the central library of the University of Kiel or at the Sektion für Spektren und Strukturdokumentation, University of Ulm (Germany).

the Technical Faculty, which gave the necessary assistance. Further I would like to thank Prof. H. Dreizler, Kiel and Dr. L. Nemes, Budapest for reading and commenting the manuscript.

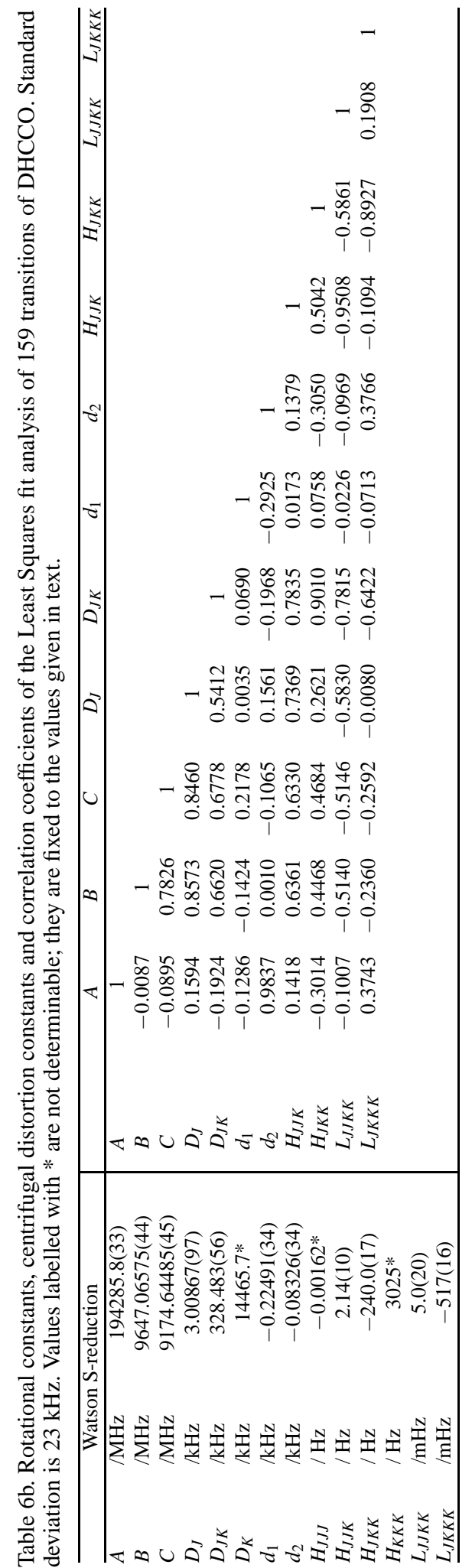


Table 7. Derived rotational constants (in MHz), moments of inertia and inertia defects (in amu $\AA^{2}$ ) for 11 isotopomers of ketene.

\begin{tabular}{lccccccc}
\hline Isotopomer & $A$ & $B$ & $C$ & $I_{a}$ & $I_{b}$ & $I_{c}$ & $\Delta$ \\
\hline $\mathrm{H}_{2} C C O^{\mathrm{a}}$ & $282101.185(409)$ & $10293.32117(80)$ & $9915.90548(82)$ & $1.7914813(27)$ & $49.0977597(43)$ & $50.9665013(42)$ & 0.077260 \\
$\mathrm{H}_{2} \mathrm{CCO}^{\mathrm{b}}$ & $282032(22)$ & $10293.31963(81)$ & $9915.90393(80)$ & $1.79192(14)$ & $49.0977759(34)$ & $50.9665184(41)$ & 0.076821 \\
$\mathrm{H}_{2} \mathrm{C}^{13} \mathrm{CO}^{\mathrm{b}}$ & $282037(26)$ & $10293.6281(11)$ & $9916.2102(11)$ & $1.79189(16)$ & $49.0963045(52)$ & $50.9649442(56)$ & 0.076750 \\
$\mathrm{H}_{2}{ }^{13} \mathrm{CCO}^{\mathrm{b}}$ & $282044(14)$ & $9960.97994(61)$ & $9607.14130(60)$ & $1.79184(10)$ & $50.7358817(31)$ & $52.6045245(33)$ & 0.076798 \\
$\mathrm{H}_{2} \mathrm{CC}^{18} \mathrm{O}^{\mathrm{b}}$ & $282002(22)$ & $9761.23954(92)$ & $9421.12530(92)$ & $1.79211(17)$ & $51.7740701(51)$ & $53.6431778(52)$ & 0.076996 \\
$\mathrm{H}_{2} \mathrm{CC}^{17} \mathrm{O}^{\mathrm{c}}$ & $282072(22)$ & $10013.4764(28)$ & $9655.9118(24)$ & $1.79167(14)$ & $50.469895(15)$ & $52.338827(14)$ & 0.077265 \\
$\mathrm{H}_{2}{ }^{13} \mathrm{C}^{13} \mathrm{CO}^{\mathrm{d}}$ & $282031(22)$ & $9960.8654(27)$ & $9607.0546(28)$ & $1.79193(14)$ & $50.736465(14)$ & $52.604999(15)$ & 0.076607 \\
$\mathrm{D}_{2} \mathrm{CCO}^{\mathrm{d}, \mathrm{e}}$ & $141490.52(88)$ & $9120.83101(34)$ & $8552.70127(35)$ & $3.571823(22)$ & $55.4093261(21)$ & $59.0899898(24)$ & 0.108849 \\
$\mathrm{D}_{2}{ }^{13} \mathrm{CCO}^{\mathrm{d}}$ & $141483.7(10)$ & $8890.4753(17)$ & $8349.8413(15)$ & $3.571995(25)$ & $56.845004(11)$ & $60.525593(11)$ & 0.108595 \\
$\mathrm{D}_{2} \mathrm{C}^{13} \mathrm{CO}^{\mathrm{d}}$ & $141484.0(14)$ & $9119.4284(24)$ & $8551.4841(23)$ & $3.571988(35)$ & $55.417848(15)$ & $59.098408(16)$ & 0.108572 \\
$\mathrm{D}_{2} \mathrm{CC}^{18} \mathrm{O}^{\mathrm{d}}$ & $141490.0(13)$ & $8641.8429(21)$ & $8130.0534(21)$ & $3.571836(33)$ & $58.480478(14)$ & $62.161843(16)$ & 0.109529 \\
$\mathrm{DHCCO}^{\mathrm{d}, \mathrm{e}}$ & $194285.8(33)$ & $9647.06578(44)$ & $9174.64485(45)$ & $2.601214(44)$ & $52.3868201(25)$ & $55.0843230(27)$ & 0.096288 \\
\hline
\end{tabular}

a [10]. Data, reported for comparison, are results of IR $+\mathrm{MW} \mathrm{fit;}{ }^{\mathrm{b}}[1] ;{ }^{\mathrm{c}}\left[{ }^{[5]}{ }^{\mathrm{d}}{ }^{\mathrm{d}}\right.$ this work; ${ }^{\mathrm{e}}$ [11]. The coefficient $505379.1 \mathrm{MHz} \cdot \mathrm{amu} \cdot \AA^{2}$ has been used for the conversion.

[1] A. Guarnieri and A. Huckauf, Z. Naturforsch. 58a, 275 (2003).

[2] L. Nemes, in: Spectroscopy: Perspectives and Frontiers (Ed. A. P. Roy), Narosa Publishing House, New Delhi, India 1997.

[3] A.L.L. East, W.D. Allen, and J. Klippenstein, J. Chem. Phys. 102, 8506 (1995).

[4] J. K. G. Watson, A. Roytburg, and W. Ulrich, J. Mol. Spectrosc. 196, 102 (1999).

[5] A. Guarnieri and A. Huckauf, Z. Naturforsch. 56a, 440 (2001).

[6] J. K. G. Watson, J. Mol. Spectrosc. 207, 16 (2001).

[7] Z. Kisiel, J. Mol. Spectrosc. 218, 58 (2003); Z. Kisiel, Strfit Program in PROSPE (Programs for ROtational SPEctroscopy). http://info.ifpan.edu.pl/ kisiel /prospe.htm.

[8] J.K. G. Watson, Aspects of Quartic and Sextic Centrifugal Effects on Rotational Energy Levels, in: Vibrational Spectra and Structure, Vol. 6 (Ed. J. R. Durig), Elsevier, Amsterdam 1977, pp. 1-89; W. Gordy and R. L. Cook, Microwave Molecular Spectra, Wiley Interscience Equations 8.111, 8.112,.8.113 and 8.117, pp. 334,335 .
[9] Program kindly delivered by Dr. Laszlo Nemes, Central Research Institute for Chemistry, Hungarian Academie of Sciences, Budapest, Hungary; Z. Kisiel, Asfit Program in PROSPE (Programs for ROtational SPEctroscopy). http://info.ifpan.edu.pl/ kisiel/prospe.htm.

[10] J. W. C. Johns, L. Nemes, K. M. T. Yamada, T. Y. Wang, J.L. Doménech, J. Santos, P. Cancio, D. Bermejo, J. Ortigoso, and R. Escribano, J. Mol. Spectrosc. 156, 501 (1992).

[11] L. Nemes and M. Winnewisser, Z. Naturforsch. 31a, 272 (1976).

[12] Š. Urban and K. M. T. Yamada, J. Mol. Spectrosc. 160, 279 (1993).

[13] F. Hegelund, J. Kauppinen, and F. Winther, Mol. Phys. 61, 261 (1987).

[14] W. Gordy and R. Cook, Microwave Molecular Spectra, Wiley Interscience, New York 1984, p. 335.

[15] J. L. Duncan, A. M. Ferguson, J. Harper, and K. H. Tonge, J. Mol. Spectrosc. 125, 196 (1987). 